\title{
Pigmented contact dermatitis to p-paraphenylenediamine in a textile factory worker
}

\author{
Mrinal Gupta
}

Treatwell Skin Centre, Opp Science College, Canal Road, Jammu, J \& K, India

Corresponding author: Dr. Mrinal Gupta, E-mail: drmrinalgupta@yahoo.com

\begin{abstract}
Pigmented contact dermatitis (PCD) is a noneczematous variant of contact dermatitis, characterized clinically by hyperpigmentation with little or no signs of dermatitis. The commonly implicated agents causing PCD include cosmetics like fragrances, lipsticks and kumkum, preservatives, optical whiteners, benzyl salicylate and metallic compounds like nickel sulphate and nickel oxide. We present a case of PCD in a 45-year old textile factory worker, who presented with gradually progressive asymptomatic hyperpigmentation of the face which started after he started working in a textile factory. Patch test revealed sensitization to $p$-Paraphenylenediamine, thereby confirming the diagnosis of PCD to p-Paraphenylenediamine.
\end{abstract}

Key words: Pigmented contact dermatitis; p-Paraphenylenediamine; occupational dermatosis; pigmented cosmetic dermatitis; textiles

\section{INTRODUCTION}

Pigmented contact dermatitis (PCD) is a noneczematous variant of contact dermatitis, characterized clinically by hyperpigmentation with little or no signs of dermatitis. PCD has been found to be caused by a large number of agents with the most commonly implicated ones being cosmetics like fragrances, lipsticks and kumkum, preservatives, optical whiteners, benzyl salicylate, minoxidil and metallic compounds like nickel sulphate, chromium hydroxide and nickel oxide [1]. Herein, we present a case of PCD in a 45-year old textile factory worker, who presented with gradually progressive asymptomatic hyperpigmentation of the face which started after he started working in a textile factory.

\section{CASE REPORT}

A 45-year-old man presented with a three year history of worsening non-pruritic pigmentation over the face. The pigmentation started over the forehead and over the period of time involved the whole face but remained asymptomatic throughout. The patient correlated the onset of lesions with his change in job, when he started working in a textile factory where he was engaged in the job of textile packing. There was no history of any similar lesions prior to that and the patient gave no history of any drug intake or hair dye use but the patient was using regular toiletries like cold creams and soap. He had no personal or family history of atopy or any similar condition in the family members. On examination, there were hyperpigmented patches, symmetrically distributed over her forehead and cheeks with relative sparing of the nose and the malar prominences (Fig. 1). Nails, mucosae, hair and rest of cutaneous examination was normal. Systemic examination and routine laboratory investigations revealed no abnormality.

Differential diagnoses considered included pigmented contact dermatitis and melasma. The clinical appearance and the patient's age were considered less typical for melasma.

Patch tests were performed with Indian standard series, cosmetic series and the patient's own products using Finn Chambers, and were read after 48 and 72 hours. 
The patch test showed positive $(2+)$ reaction to p-Paraphenylenediamine (PPD)(Fig. 2). Other allergens and patient's own products showed negative response.

On the basis of history, clinical examination and patch test, a diagnosis of PCD to PPD was made. The patient was advised a change in job profile which he refused and was started on topical $4 \%$ hydroquinone cream at night with sunscreens during daytime. After three months of therapy, there was only a mild improvement in symptoms.

\section{DISCUSSION}

The term "pigmented contact dermatitis" was coined by Osmundsen in 1970, who described an epidemic of melanosis in Copenhagen which was due to contact

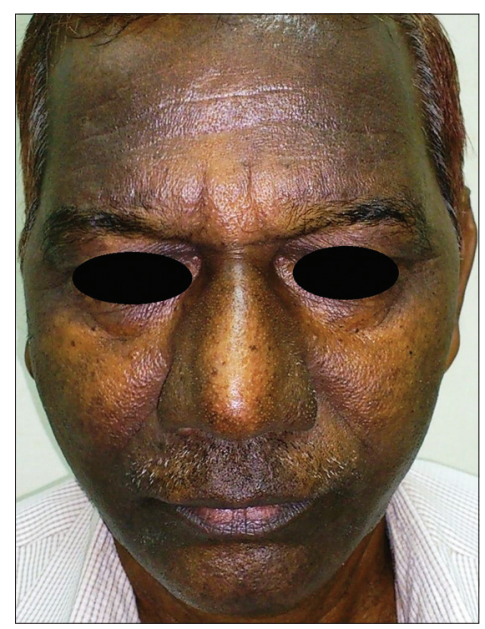

Figure 1: Hyperpigmented patches on the temporal, forehead and zygomatic areas, with sparing of the nose and malar prominences.

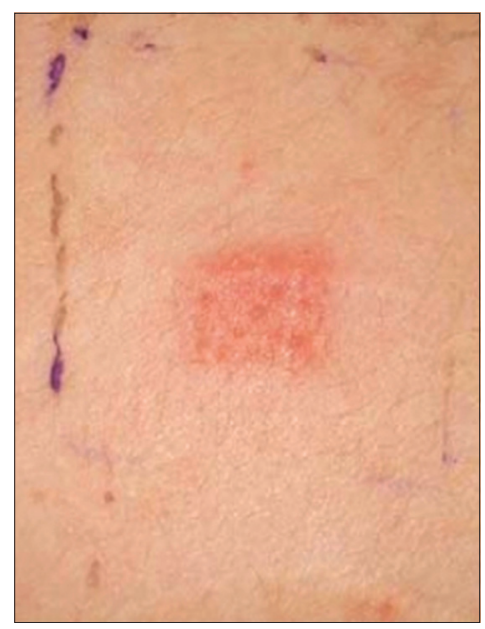

Figure 2: Patch test positive reaction to PPD. dermatitis caused by an optical whitener present in a washing powder [2]. A large number of chemicals with a similar tendency to induce PCD have been identified in the subsequent years. The exact mechanism by which these chemicals induce pigmentation is unknown but it has been postulated that these agents produce a cytolytic type of type IV allergy mainly at the basal layer of the epidermis that results in pigmentary incontinence [3].

Clinically, PCD manifests as reticulate reddish brown or slate grey pigmentation without any signs of preceding inflammation like itching, erythema or scaling. PCD usually occurs due to direct contact with the allergens and the sites coming in contact with the causative agents are commonly affected with face being the most commonly involved site. Diagnosis is mainly done on clinical examination but patch test has been found useful in confirmation of the diagnosis [1]. Patch testing should be carried out with standard series, cosmetic series, fragrance series and the personal products of the patients as it helps in identification of the causative agent. In an Israeli study, 26 patients with PCD were subjected to patch test and the most commonly identified allergens were nickel sulphate, fragrance mix and potassium dichromate [4].

PPD, an arylamine derivative first described by Hoffmann in 1863, is one of the most commonly implicated agents causing contact sensitivity. It is a commonly used coloring agent and is present in hair dyes, dyes for henna tattoos, textiles, leather and fur, and black rubbers. Being a member of 1,4-substituted benzenes, it cross-reacts with para-amino benzoic acid (PABA), sulphonamides, para-amino salicylic acid, ester anesthetics, thiazides, sesquiterpene-lactone mix, and azo dyes [5]. The reported prevalence of positive patch test reactions to PPD among dermatitis patients is $4.4 \%$ in Asia, $4.1 \%$ in Europe, $6.0 \%$ in North America, and $11.5 \%$ in India. The most common clinical presentations of PPD sensitivity include contact dermatitis localized to sites of contact or photoexposed skin, periorbital dermatitis, airborne contact dermatitis, hand dermatitis and pigmented contact dermatitis [6].

Contact sensitivity from PPD is becoming a prevalent health problem. Strict regulations are required to regulate its concentration in various products. As it is used in a large number of industries, it can pose a risk for various occupational groups as well as the general public. There needs to be a continued awareness among 
www.odermatol.com

the factory workers as well as the general population of the potential risk of PPD, especially in darker skin types where the pigmentary changes can be quite marked.

\section{REFERENCES}

1. Shenoi SD, Rao R. Pigmented contact dermatitis. Indian J Dermatol Venereol Leprol. 2007;73:285-7.

2. Osmundsen PE. Pigmented contact dermatitis. Br J Dermatol. 1970;83:296-301.

3. Nakayama H, Matsuo S, Hayakawa K, Takashi K, Shigematsu T, Ota S. Pigmented cosmetic dermatitis. Int J Dermatol. 1984;23:299-305.

4. Trattner A, Hodak E, David M. Screening patch tests for Pigmented contact dermatitis in Israel. Contact Dermatitis. 1999;40:155-7.

5. Xie Z, Hayakawa R, Sugiura M, Kojima H, Konishi H, Ichihara G, Takeuchi Y, et al. Experimental study on skin sensitization potencies and cross-reactivities of hair-dye-related chemicals in guinea pigs. Contact Dermatitis. 2000;42:270-5.

6. Gupta M, Mahajan VK, Mehta KS, Chauhan PS. Hair dye dermatitis and p-phenylenediamine contact sensitivity: A preliminary report. Indian Dermatol Online J. 2015;6:241-5.

Copyright by Mrinal Gupta. This is an open-access article distributed under the terms of the Creative Commons Attribution License, which permits unrestricted use, distribution, and reproduction in any medium, provided the original author and source are credited.

Source of Support: Nil, Conflict of Interest: None declared. 